\title{
Similar early clinical presentations in familial and non- familial frontotemporal dementia
}

\author{
O Piguet, W S Brooks, G M Halliday, P R Schofield, P M Stanford, J B J Kwok, M-G Spillantini, \\ D Yancopoulou, P J Nestor, G A Broe, J R Hodges
}

J Neurol Neurosurg Psychiatry 2004;75:1743-1745. doi: 10.1136/jnnp.2003.031948

Background: It is unclear whether there are early clinical features that can distinguish between patients with familial and non-familial frontotemporal dementia (FTD).

Objective: To compare the clinical features of FTD cases who have tau gene mutations with those of cases with a family history of FTD but no tau gene mutation, and with sporadic cases with neither feature.

Methods and results: Comparisons of the behavioural, cognitive, and motor features in 32 FTD patients (five positive for tau gene mutations, nine familial but tau negative, and 18 tau negative sporadic) showed that age of onset and duration to diagnosis did not differ between the groups. Apathy was not observed in tau mutation positive cases, and dysexecutive signs were more frequent in familial tau mutation negative cases. Memory deficits and behavioural changes were common in all groups.

Conclusions: In comparison with other neurodegenerative conditions such as Alzheimer's disease and Parkinson's disease, neither tau gene mutations nor strong familial associations confer earlier disease susceptibility.

$\mathrm{F}$ rontotemporal dementia (FTD) is the second most common form of early onset dementia after Alzheimer's disease and accounts for up to $20 \%$ of all cases in the under-65s. ${ }^{1}$ It is characterised by gross frontotemporal atrophy with variable underlying histopathology. ${ }^{2}$ Up to $45 \%$ of all FTD cases show a familial pattern of inheritance, with mutations in the tau gene on chromosome 17 associated with the disease. ${ }^{3}$ Studies have also shown linkage to chromosomes 3 and $9 .{ }^{4}$ Clinically FTD is characterised by progressive changes in behaviour and personality, or progressive aphasia. ${ }^{5}$ On neuropsychological tests, FTD patients show executive dysfunction that is disproportionate compared with other cognitive domains such as memory or visuoconstructive abilities.

Cases with familial FTD can present with various extrapyramidal disorders in addition to behavioural changes. ${ }^{6}$ While this suggests that familial FTD may differ clinically from sporadic FTD, a recent study reported identical clinical presentations in 22 FTD families, half with tau gene mutations. ${ }^{7}$ In familial Alzheimer's disease or Parkinson's disease, there is a significant shift to an earlier age of onset, and often a more rapid progression of disease. ${ }^{8}$ For FTD, the genetic contribution to the clinical presentation, age of onset, and rate of progression remains unclear.

In this study, we compared the clinical features of FTD cases who had tau gene mutations with those of cases with a family history of FTD but no tau gene mutation, and with sporadic cases with neither of these features.

\section{METHODS}

The majority of FTD cases (26 of 32, 80\%) were recruited from specialist tertiary referral dementia clinics in Cambridge and Sydney, and the remainder from case referral. Recruitment for research was approved by the human ethics committee of the University of Sydney and the Addenbrooke's Hospital local ethics committee. Patients were examined clinically by experienced geriatricians or behavioural neurologists, had their cognitive function assessed, and were selected for inclusion using the Lund and Manchester criteria for FTD. ${ }^{9}$ Fourteen affected probands with autosomal dominant FTD (two or more affected individuals over two generations) and 18 apparent sporadic FTD cases (seven from Australia, 11 from Cambridge) were selected. Sporadic FTD cases were selected on the basis of an informative, but negative, family history; both parents had lived beyond 60 with no evidence of dementia and no other affected family members. All cases, familial or sporadic, were screened for tau mutations as previously reported..$^{11}$ Patients were allocated to one of three groups:

- patients with an identified tau gene mutation (tau mutation positive cases);

- patients with a positive family history but without an identified tau gene mutation (familial tau mutation negative cases);

- non-familial, tau negative, clinically diagnosed FTD patients (sporadic cases).

Ten cases died during the study period, and neuropathology was available on nine (two tau mutation positive, five familial tau mutation negative, and two sporadic), confirming FTD in all cases (table 1).

For each patient, a retrospective analysis of clinical characteristics within 12 months of initial presentation was assessed from the available information (comprehensive medical files were available and in most instances contained interview notes with patient, neuropsychological test results, and informant interview). These included age of onset, duration between onset of symptoms and diagnosis, and presence of behavioural, cognitive, or motor changes. For many cases information from either a behavioural questionnaire developed in Cambridge $\mathrm{e}^{13}$ or the neurospychiatric inventory (NPI) $)^{14}$ were available. Behavioural changes comprised major disinhibition, impulsivity, stereotypical behaviours, or change in eating patterns. Apathy was recorded as a separate feature. Cognitive changes included dysexecutive symptoms (for example, poor planning and organisational skills or concreteness of ideation), impaired memory (impaired new learning or temporal disorientation), and language deficits (fluent or non-fluent type). Motor symptoms included extrapyramidal features and apraxia. When relevant, age of death and disease duration were also recorded. 
Table 1 Demographic and clinical characteristics at presentation for the tau mutation positive, familial tau mutation negative, and sporadic FTD patients

\begin{tabular}{|c|c|c|c|}
\hline & $\begin{array}{l}\text { Tau mutation } \\
\text { positive }^{*}(n=5)\end{array}$ & $\begin{array}{l}\text { Familial tau mutation } \\
\text { negative }(n=9)\end{array}$ & Sporadic $(n=18)$ \\
\hline \multicolumn{4}{|l|}{ Demographic information } \\
\hline $\operatorname{Sex}(F / M)$ & $1 / 4$ & $5 / 4$ & $4 / 14$ \\
\hline Age of onset (years)†‡ & $50(4.8), 43$ to 55 & $57(8.5), 44$ to 71 & $52(10), 33$ to 73 \\
\hline Symptom duration to diagnosis (years) $† \ddagger$ & $1.4(0.5), 1$ to 2 & $3.7(3.2), 1$ to 10 & $4.1(3.0), 1$ to 10 \\
\hline Deceased & 2 & 5 & 2 \\
\hline \multicolumn{4}{|l|}{ Symptom presentations (n (\%)) } \\
\hline Behaviour & $5(100)$ & $8(89)$ & $12(67)$ \\
\hline Apathy & $0 \S(0)$ & $7(78)$ & $10(56)$ \\
\hline Dysexecutive & $0(0)$ & $7 \S(87)$ & $6(33)$ \\
\hline Language & $1(20)$ & $1(11)$ & $8(44)$ \\
\hline Memory & $3(60)$ & $7(78)$ & $7(39)$ \\
\hline Apraxia & $1(20)$ & $1(11)$ & $0(0)$ \\
\hline Extrapyramidal & $1(20)$ & $1(11)$ & $3(17)$ \\
\hline Major psychiatric disorder & $0(0)$ & $0(0)$ & 6 (33) \\
\hline \multicolumn{4}{|c|}{$\begin{array}{l}\text { *Mutations identified were all within or flanking exon } 10: \mathrm{S} 305 \mathrm{~S}^{11} \mathrm{P} 301 \mathrm{~L} \text { (unpublished), } 10+16 \text { (unpublished) } \\
10+19,{ }^{12} \text { and } 10+29 .{ }^{12} \\
+ \text { Values are mean (SD), range. } \\
\text { †Non-significant differences between groups. } \\
\text { §Information not available for one case. }\end{array}$} \\
\hline
\end{tabular}

Non-parametric Kruskall-Wallis tests were used to determine differences between groups in age and durations, and $\chi^{2}$ tests to determine differences in the clinical features. The level of significance was set at $\mathrm{p}<0.05$.

\section{RESULTS}

Demographic and clinical information for the three case groups is given in table 1 . There were no significant differences between the FTD groups in age of onset or time between disease onset and diagnosis ascertainment (table 1). For the cases coming to necropsy, disease duration averaged nine years but was very variable in the familial tau mutation negative group, ranging from one to 13 years.

At presentation, motor signs were infrequent and behavioural changes were very common in all three FTD groups (table 1). Apathy was not present in the tau mutation positive group but was commonly reported in the familial tau mutation negative and sporadic cases, this difference being significant $\left(\chi^{2}=6.7, p=0.034\right.$; table 1$)$. The familial tau mutation negative group showed dysexecutive symptoms more often than the other groups $\left(\chi^{2}=10.9, \mathrm{p}=0.004\right.$; table 1). Major psychiatric disorders-such as depression, paranoid psychosis, delusions and hallucinations-were reported at presentation in the sporadic group only, and these disturbances were severe enough to require admission to a mental health facility in three cases. This difference in feature presentation failed to reach statistical significance $(\mathrm{p}=0.056)$.

\section{DISCUSSION}

Our most surprising finding was the lack of significant difference in age of disease onset between cases with familial and sporadic FTD, and between the tau mutation positive and tau mutation negative familial cases, although the range of ages and duration of symptoms is greater for tau mutation negative and sporadic cases. This contrasts dramatically with the earlier age of onset and more rapid disease progression observed in familial Alzheimer's disease. ${ }^{8}$ Similar mean ages of disease onset (and ranges) have been reported before in well defined familial and sporadic FTD groups. ${ }^{15}$ These findings suggest that one or more genes could influence the age of disease onset and the duration of illness in these different FTD groups. In tau positive FTD cases, the genetic contribution is mostly through the tau gene mutation. In the other FTD cases, the effect may possibly be oligogenic, in that a small number of risk factor alleles, on several genes, may show convergence and produce a similar effect. These alleles would remain individually neither necessary nor sufficient for the development of the disease and therefore would not show linkage to other loci. In all FTD cases, the variation in disease onset and duration would reflect the dose effect and contribution of these alleles to the disease. The larger ranges of age of onset and duration of symptoms to diagnosis observed in the familial tau mutation negative and sporadic FTD groups in this study, as well as in tau positive families affected by the same mutation, ${ }^{36}$ would give support to this position. In all instances, additional environmental contributions cannot be discounted.

The lack of differences in age of disease onset and duration of symptoms before diagnosis in our study is unlikely to be explained by group sizes. Recent studies on familial (tau mutation positive or other familial) and sporadic FTD have reported very similar ranges of onset and durations, and a wide variability both within and between tau positive families affected by the same gene mutation. ${ }^{36}$ For our dataset, power calculations indicated that our group sizes gave us $82-88 \%$ power to detect a between-group difference in onset age that was half the size reported for other familial dementias. The genetic contribution to the onset and course of the disease may be more potent in FTD than in Alzheimer's disease or Parkinson's disease, as the average onset for FTD was before 60 , whereas the majority of other neurodegenerative diseases has an average onset after 60 (but see other reports $\left.{ }^{816}\right)$.

Clinical features expected to differentiate the tau mutation positive FTD group were not observed in our cohort. In particular, extrapyramidal signs were rare in all FTD groups. Although not regarded as a hallmark of FTD, memory disturbance was common, as recently reported.${ }^{17}$ Prominent behavioural change was by far the most common clinical presentation for all FTD cases, possibly reflecting the selection criteria for this study. We found that dysexecutive symptoms were a more common presenting feature in familial tau mutation negative FTD cases. This may be because comparisons were made with longstanding executive deficits in tau mutation positive cases $^{18}$ as opposed to emerging executive deficits in familial tau mutation negative cases. In contrast, schizophreniform symptoms in association with frontal features have been described in only one family with a tau gene mutation showing amygdala involvement. ${ }^{19}$ 
The pattern of clinical features did not vary after considering the source of the cases (Australia versus the UK), or after applying more stringent selection criteria for the familial tau negative cases (that is, three instead of two members over two generations).

This study shows that the clinical expression of FTD is relatively uniform early in the disease process, with some exceptions, despite clearly identified different underlying biological correlates. Different genetic mechanisms could explain this convergence in the clinical presentation across groups. These findings warrant further examination of larger FTD cohorts for a better understanding of the clinical expression of the various forms of this disease as well as their associated genetic processes.

\section{ACKNOWLEDGEMENTS}

This research was supported in part by the National Health and Medical Research Council of Australia block grant (993050), network grant (983302), research fellowships (157209, 157212) and project grants (113804, 157034); a University of New South Wales Deans scholarship; the Sir Edward Dunlop Medical Research Foundation; an MRC studentship and an MRC programme grant from the Medical Research Council of the UK.

\section{Authors' affiliations}

O Piguet, W S Brooks, G A Broe, G M Halliday, Prince of Wales Medical Research Institute, University of New South Wales, Sydney, New South Wales, Australia

P R Schofield, P M Stanford, J B J Kwok, The Garvan Institute for Medical Research, University of New South Wales, Sydney, New South Wales, Australia

M-G Spillantini, D Yancopoulou, Centre for Brain Repair, University Department of Neurology, Addenbrooke's Hospital, Cambridge, UK P J Nestor, J R Hodges, MRC Cognition and Brain Sciences Unit, University Department of Neurology, Addenbrooke's Hospital, Cambridge, UK

Competing interests: none declared

Correspondence to: Professor G Halliday, Prince of Wales Medical Research Institute, Barker Street, Randwick, NSW 2031, Australia; G.Halliday@unsw.edu.au

Received 6 November 2003

In revised form 19 February 2004

Accepted 22 March 2004

\section{REFERENCES}

1 Ratnavalli E, Brayne C, Dawson K, et al. The prevalence of frontotemporal dementia. Neurology 2002;58:1615-21.

2 Jackson $M$, Lowe J. The new neuropathology of degenerative frontotemporal dementias. Acta Neuropathol (Berl) 1996:91:127-34.

3 Reed LA, Wszolek ZK, Hutton M. Phenotypic correlations in FTDP-17. Neurobiol Aging 2001;22:89-107.

4 Hosler BA, Siddique T, Sapp PC, et al. Linkage of familial amyotrophic lateral sclerosis with frontotemporal dementia to chromosome 9q21-q22. JAMA 2000;284:1664-9

5 Grossman M. Frontotemporal dementia: a review. J Int Neuropsychol Soc 2002;8:566-83.

6 Janssen JC, Warrington EK, Morris HR, et al. Clinical features of frontotemporal dementia due to the intronic tau 10(+16) mutation. Neurology 2002;58:1161-8.

7 Morris HR, Khan MN, Janssen JC, et al. The genetic and pathological classification of familial frontotemporal dementia. Arch Neurol 2001;58:1813-16.

8 Wisniewski T, Dowjat WK, Buxbaum JD, et al. A novel Polish presenilin-1 mutation (P1 1 ZL) is associated with familial Alzheimer's disease and leads to death as early as the age of 28 years. Neuroreport 1998;9:217-21.

9 Lund and Manchester Groups. Clinical and neuropathological criteria for frontotemporal dementia. J Neurol Neurosurg Psychiatry 1994:57:416-18.

10 Rizzini C, Goedert M, Hodges JR, et al. Tau gene mutation K257T causes a tauopathy similar to Pick's disease. J Neuropathol Exp Neurol 2000;59:990-1001.

11 Stanford PM, Halliday GM, Brooks WS, et al. Progressive supranuclear palsy pathology caused by a novel silent mutation in exon 10 of the tau gene: expansion of the disease phenotype caused by tau gene mutations. Brain 2000;123:880-93.

12 Stanford PM, Shepherd CE, Halliday GM, et al. Mutations in the tau gene that cause an increase in 3 repeat tau and frontotemporal dementia. Brain 2003;126:814-26.

13 Bozeat S, Gregory CA, Ralph MA, et al. Which neuropsychiatric and behavioural features distinguish frontal and temporal variants of frontotemporal dementia from Alzheimer's disease? I Neurol Neurosurg Psychiatry 2000;69:178-86.

14 Cummings JL, Mega M, Gray K, et al. The Neuropsychiatric Inventory: comprehensive assessment of psychopathology in dementia. Neurology 1994;44:2308-14.

15 Houlden H, Baker M, Adamson J, et al. Frequency of tau mutations in three series of non-Alzheimer's degenerative dementia. Ann Neurol 1999:46:243-8.

16 Alzheimer's Disease Collaborative Group. The structure of the presenilin 1 (S182) gene and identification of six novel mutations in early onset $A D$ families. Alzheimer's Disease Collaborative Group. Nat Genet 1995; 11:219-20.

17 Rosen HJ, Hartikainen KM, Jagust W, et al. Utility of clinical criteria in differentiating frontotemporal lobar degeneration (FTLD) from AD. Neurology 2002;58:1608-15.

18 Geschwind DH, Robidoux J, Alarcon M, et al. Dementia and neurodevelopmental predisposition: cognitive dysfunction in presymptomatic subjects precedes dementia by decades in frontotemporal dementia. Ann Neurol 2001;50:741-6.

19 Poorkaj P, Bird TD, Wijsman E, et al. Tau is a candidate gene for chromosome 17 frontotemporal dementia. [erratum appears in Ann Neurol 1998 Sep;44(3):428]. Ann Neurol 1998;43:815-25. 